If the continuous aponeurotic or deep fascia-suture could be put on the slack or relieved from all strain and to merely act as an apposition suture, this would secure an ideal condition for wound healing. The figure-of-eight or throughand-through sutures fall short of this ideal, as they take in a very limited area and act mainly as reenforcing sutures at the spot where inserted.

For some time I have been using with great satisfaction and a sense of security what might be called crossed mattresssutures. They take in a large area of the aponeurosis, and taking the stain off from the continuous suture, act as tiue tension sutures.

Another desirable action of this suture is: As it firmly holds together the cut edges of the subcutaneous tissue and skin during healing, no ugly, broad scar results, but only a faint streak.

It is quickly placed as follows: Penetrate skin and subcutaneous tissue at $a$ in illustration, emerge in front of aponeurosis at $b$, cross over in front of suture line and penetrate aponeurosis at $c$, emerge at $d$, cross over in front of suture
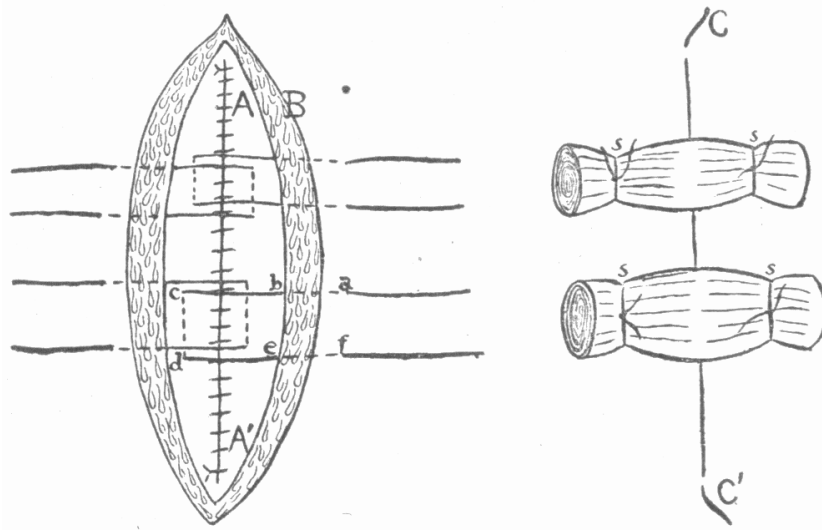

Diagram showing method of placing tension sutures. $A$, aponeurosis; $B$, skin and fat $A, A^{\prime}$, continuous suture of aponeurosis; $a, b, c, d, e, f$, points of entrance and emergence of crossed mattress-sutures; $C, C^{\prime}$, subcuticular suture ; $8, s$, tension sutures tied over rolls of gauze.

line and penetrate subcutaneous tissue at $e$, emerge through skin at $f$. The opposite suture is placed in a similar manner. Two or three pairs are used. After the subcuticular or continuous horsehair suture is taken, the crossed mattress-sutures are tied over rolls of gauze. They are readily removed by extting one limb of each suture close to the skin.

\section{THE PREPARATION OF DRIED "ZYMASE" FROM YEAST, FOR USE IN FERMENTATION} TESTS OF URINE *

\section{J acob Rosenbloom, M.D.,Ph.D., Pittsburgh, Pa.}

On account of the well-known fact that, by fermentation with yeast, glucose is decomposed into alcohol and carbon dioxid, we have at present the excellent fermentation test for glucose. This method is not only valuable for qualitative purposes to distinguish the kind of reducing substance present in the urine but is of great use for the quantitative estimation of glucose.

The method has one disadvantage from the necessity of using perfectly fresh yeast in its performance. I have, however, devised a method to isolate the enzyme from the yeast in a dried condition and have found that the enzyme in this state is still active five months ${ }^{1}$ after its preparation.

Grind up thoroughly in a mortar three cakes of compressed yeast with about 200 c.c. of water and $10 \mathrm{gm}$. of sand. Press this fluid through cheese-cloth and add the expressed liquid, with constant stirring, to about five times' its volume of 95

* From the Blochemical Laboratory of the Western Pennsylvania Hospital, Pittsburgh, Pa.

1. It is possible that I shall find that this preparation will be active for a much longer time, but as five months have passed demonstrated. per cent. alcohol. Allow the precipitate to settle, filter and wash the precipitate on the filter-paper with about 50 c.c. of alcohol followed by 50 c.c. of ether. Allow the precipitate to dry in the air after spreading it on a glass plate. Powder and put residue in a tightly corked amber bottle. To use this powder to ferment urine, take about as much as the end of a medium-sized knife blade will hold, mix it thoroughly with the urine to be tested and pour the mixture into a fermentation tube.

I have found that certain well-known conditions are not observed by many workers when using the fermentation test, so it may be of some service to call attention to these details.

1. The urine to be tested, freed from protein if found present, must previously be slightly acidulated with 1 per cent. tartaric or acetic acid solution. This lessens the possibility of bacterial fermentation; also some of the carbon dioxid of the urine may have chemically combined with the monohydrogen sodium phosphate that is present, forming dihydrogen sodium phosphate and sodium bicarbonate. This source of possible error is avoided by the acidulation which converts the monohydrogen salt into the dihydrogen salt.

2. The urine should be boiled after acidulating to dislodge any combined carbon dioxid.

3. Besides the fermentation test applied to the urine, two control tests should be made, the one with normal urine to which a little glucose is added to prove the activity of the yeast, and another of normal urine alone, to prove by the absence of gas, that there is no self-fermentation of the yeast. With these points in mind it will be found that the fermenta. tion test can always be relied on and will not be found wanting.

5659 Beacon Street.

\section{Therapeutics}

\section{PROTEIN POISONING-ANAPHYLAXIS-URTICARIA}

\section{PROTEIN POISONING}

The fact that bacteria could cause protein poisoning was first noted and the condition described, in 1903, by Victor C. Vaughan of Ann Arbor. Protein poisoning is the cause of most urticarial conditions, of many of the skin eruptions, of many of the simple, so-called febricula (a name applied to a fever lasting one or two days with no positive diagnosis determinable), and ali of these disturbances are really forms of anaphylaxis.

Some protein poisons may cause a lowered or subnormal temperature rather than fever. This is apparently due to a marked dilatation of the peripheral blondvessels, especially of the splanchnic area, similar to that in shock. With other protein poisonings there may be, for several days, an irregular temperature with morning remissions. If such poisonings persist and the toxins are not rapidly expelled, neutralized or destroyed, there will be an increased elimination of nitrogen and a progressive loss of weight.

A scientific discussion of these protein poisonings has recently been presented by Vaughan, ${ }^{1}$ and should be read by every physician, as a thorough understanding of the causes of these disturbances of the blood and nervous system will lead to proper treatment.

The symptoms of many diseases are due to the so-called "parenteral" digestion of proteins. Hay-fever: and paroxysms of asthma are caused in sensitive individuals by the pollen of different plants, the emanations from different animals, or the dust or odors of many kinds of substances. Any susceptible individual may be sensitized, so to speak, by one or more of these irritant causes and not by others. The inhalation of some

1. Vaughan Viator $C$. The Protein Poison and Its Rolation to Disease, 'TIn Jocrsat A. M. A., Nov. 15, 1913, p. 1761. 